\title{
UCRL-TR-233699
}

LAW RENCE LIVERMORE N A T IO N A L LABORATORY
MatProps: Material Properties

Database and Associated Access Library

J. K. Durrenberger, R. C. Becker, D. M. Goto, J.

R. Neely, B. K. Wallin

August 15, 2007 
This document was prepared as an account of work sponsored by an agency of the United States Government. Neither the United States Government nor the University of California nor any of their employees, makes any warranty, express or implied, or assumes any legal liability or responsibility for the accuracy, completeness, or usefulness of any information, apparatus, product, or process disclosed, or represents that its use would not infringe privately owned rights. Reference herein to any specific commercial product, process, or service by trade name, trademark, manufacturer, or otherwise, does not necessarily constitute or imply its endorsement, recommendation, or favoring by the United States Government or the University of California. The views and opinions of authors expressed herein do not necessarily state or reflect those of the United States Government or the University of California, and shall not be used for advertising or product endorsement purposes.

This work was performed under the auspices of the U.S. Department of Energy by University of California, Lawrence Livermore National Laboratory under Contract W-7405-Eng-48. 


\title{
MatProp: An XML Material Property Database and Associated Access Library (Version 1.0)
}

\author{
Kevin Durrenberger*, Richard Becker, Dana Goto, Rob Neely, Brad Wallin \\ Lawrence Livermore National Laboratory \\ August 2007
}

Coefficients for analytic constitutive and equation of state models (EOS), which are used by many hydro codes at LLNL, are currently stored in a legacy material database (Steinberg, UCRL-MA-106349). Parameters for numerous materials are available through this database, and include Steinberg-Guinan and Steinberg-Lund constitutive models for metals, JWL equations of state for high explosives, and Mie-Gruniesen equations of state for metals. These constitutive models are used in most of the simulations done by ASC codes today at Livermore. Analytic EOSs are also still used, but have been superseded in many cases by tabular representations in LEOS (http://leos.llnl.gov).

Numerous advanced constitutive models have been developed and implemented into ASC codes over the past 20 years. These newer models have more physics and better representations of material strength properties than their predecessors, and therefore more model coefficients. However, a material database of these coefficients is not readily available. Therefore incorporating these coefficients with those of the legacy models into a portable database that could be shared amongst codes would be most welcome. The goal of this paper is to describe the MatProp effort at LLNL to create such a database and associated access library that could be used by codes throughout the DOE complex and beyond.

We have written an initial version of the MatProp database and access library and our DOE/ASC code ALE3D (Nichols et. al., UCRL-MA-152204) is able to import information from the database. The database, a link to which exists on the Sourceforge server at LLNL, contains coefficients for many materials and models (see Appendix), and includes material parameters in the following categories - flow stress, shear modulus, strength, damage, and equation of state.

Future versions of the Matprop database and access library will include the ability to read and write material descriptions that can be exchanged between codes. It will also include an ability to do unit changes, i.e. have the library return parameters in user-specified unit systems. In addition to these, additional material categories can be added (e.g., phase change kinetics, etc.).

The Matprop database and access library is part of a larger set of tools used at LLNL for assessing material model behavior. One of these is MSlib, a shared constitutive material model library. Another is the Material Strength Database (MSD), which allows users to compare parameter fits for specific constitutive models to available experimental data. Together with Matprop, these tools create a suite of capabilities that provide state-of-the-art models and parameters for those models to integrated simulation codes.

This document is broken into several appendices. Appendix A contains a code example to retrieve several material coefficients. Appendix B contains the API for the Matprop data access library. Appendix $\mathrm{C}$ contains contains a list of the material names and model types currently 
available in the Matprop database. Appendix D contains a list of the parameter names for the currently recognized model types. Appendix E contains a full xml description of the material Tantalum.

For additional information about Matprop, email Kevin Durrenberger at durrenberger1@llnl.gov 


\section{Appendix A: Code examples}

Following are some examples of how material is accessed via the MatProp C++ API (a C/Fortran API is planned for the future). The underlying file format (XML) is invisible to the client. Many of these examples build on the ones prior

- Open a database of standard shared materials, access the material Tantalum

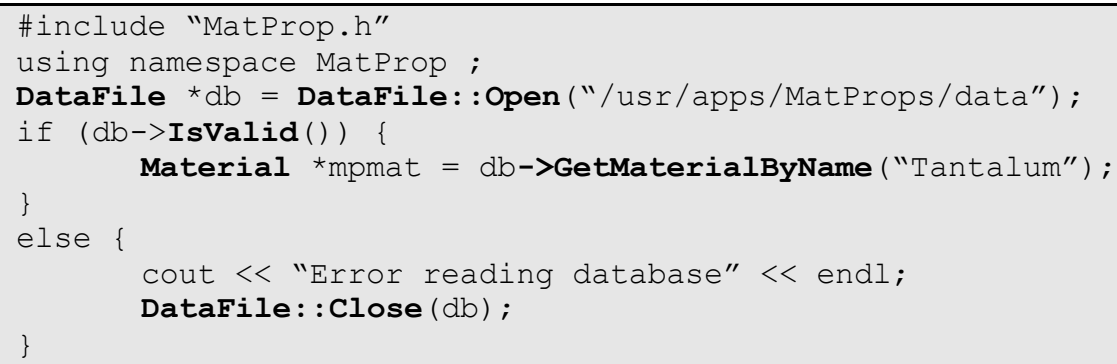

- Given a material, read some of the material properties

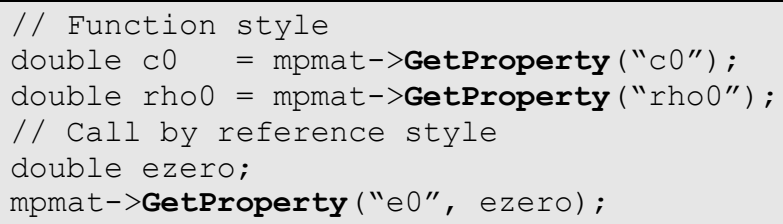

- Access a material using an identifier from a legacy database.

// Access material "25" using steinberg-guinan ID

Material *sgeos_mat $=\mathrm{db}->$ GetMaterialByLegacyId(MatProp::SGEOS, 25)

- Select the EOS model from that material and begin querying its parameters

EOSModel *mpeos = sgeos_mat->SetEOSModel ("mie_gruniesen");

formNum = mpeos->GetIntParameter ("eosform");

switch (formNum) \{

...

- Select the standard "blessed" types for a given material model, and load all of the parameters for that model

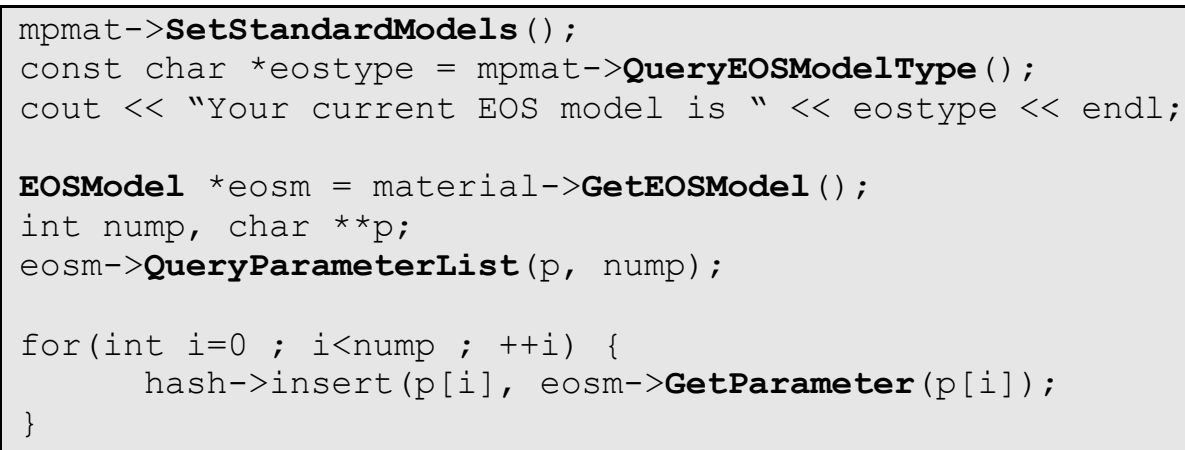




\section{Appendix B: Matprop data access library API}

\section{MatProp::DataFile Class Reference}

The DataFile class provides an interface for accessing a MatProp "database" (which could be a single monolithic XML file, or a collection of files - one-per-material.

\begin{tabular}{|c|c|}
\hline \multicolumn{2}{|c|}{ Public Member Functions } \\
\hline bool & $\begin{array}{l}\text { IsValid () const } \\
\text { True if the database was successfully read in. }\end{array}$ \\
\hline void & $\begin{array}{l}\text { SetUnits (Units units) } \\
\text { Allows the user to specify which units they prefer requests returned in. }\end{array}$ \\
\hline void & $\begin{array}{l}\text { SetVersion (const char *version) } \\
\text { Set the version of data to retrieve. }\end{array}$ \\
\hline void & $\begin{array}{l}\text { Write (const char *path) } \\
\text { Writes out a database. }\end{array}$ \\
\hline Material * & $\begin{array}{l}\text { GetMaterialByName (const char *materialName) } \\
\text { Get a material by full name of the material. }\end{array}$ \\
\hline Material * & $\begin{array}{l}\text { GetMaterialByLegacyld (LEGACYID, const char *) } \\
\text { Get Material by the legacy ID. }\end{array}$ \\
\hline \multicolumn{2}{|c|}{ Static Public Member Functions } \\
\hline DataFile * & $\begin{array}{l}\text { Open (const char *) } \\
\text { Static method for loading a default DataBase in a specific directory. }\end{array}$ \\
\hline DataFile * & $\begin{array}{l}\text { Open (const char *, const char *) } \\
\text { Static method for loading a DataBase by giving the file name and directory } \\
\text { path. }\end{array}$ \\
\hline void & $\begin{array}{l}\text { Close (DataFile *db) } \\
\text { Use this function in lieu of the destructor Closes out a database and } \\
\text { destroys the object. }\end{array}$ \\
\hline
\end{tabular}

\section{MatProp::Material Class Reference}

A Material is a collection of models, which together defines a consistent material model.

Public Member Functions

$\begin{array}{ll} & \text { Material (XNode *materialRoot) } \\ & \text { Constructor. } \\ & \text { Material () } \\ & \text { Destructor. } \\ & \text { GetName () const } \\ & \text { Return the name of the material. } \\ \text { const char * }{ }^{*} & \text { FreeName (const char *matname) const } \\ & \text { Free the memory allocated by the GetName() call. } \\ & \text { QueryPropertyList (char ** \&properties, int \&numProperties) } \\ & \text { Return a list of the property names in this material. }\end{array}$




\begin{tabular}{|c|c|}
\hline void & $\begin{array}{l}\text { FreePropertyList (char }{ }^{* *} \text { \&properties) const } \\
\text { Free resources (memory) associated with the propery list query. }\end{array}$ \\
\hline double & $\begin{array}{l}\text { GetProperty (const char *propertyName) const } \\
\text { Get a property parameter of a material. }\end{array}$ \\
\hline int & $\begin{array}{l}\text { GetIntProperty (const char *propertyName) const } \\
\text { Get an integer property parameter of a material. }\end{array}$ \\
\hline void & $\begin{array}{l}\text { GetProperty (const char *propertyName, double \&value) const } \\
\text { Get a property parameter of a material. }\end{array}$ \\
\hline void & $\begin{array}{l}\text { GetIntProperty (const char *propertyName, int \&value) const } \\
\text { Get an integer property parameter of a material. }\end{array}$ \\
\hline void & $\begin{array}{l}\text { SetStandardModels (const char *shortcutName) } \\
\text { Specify a standard set of models for this material based on one of the } \\
\text { "shortcuts". }\end{array}$ \\
\hline ModelBase * & $\begin{array}{l}\text { SetModel (const char *modelType, const char *modelName) } \\
\text { Specify the type of model based on the modelType and modelName. }\end{array}$ \\
\hline EOSModel * & $\begin{array}{l}\text { SetEOSModel (const char *modelName) } \\
\text { Specify the type of EOS (equation of state) model that this material should } \\
\text { use. }\end{array}$ \\
\hline EOSModel * & $\begin{array}{l}\text { GetEOSModel () } \\
\text { Return a pointer to the EOS model for this material. }\end{array}$ \\
\hline FlowStressModel * & $\begin{array}{l}\text { SetFlowStressModel (const char *modelName) } \\
\text { Specify the type of flow stress model that this material should use. }\end{array}$ \\
\hline FlowStressModel * & $\begin{array}{l}\text { GetFlowStressModel () } \\
\text { Return a pointer to the flow stress model for this material. }\end{array}$ \\
\hline DamageModel * & $\begin{array}{l}\text { SetDamageModel (const char *modelName) } \\
\text { Specify the type of damage model that this material should use. }\end{array}$ \\
\hline DamageModel * & $\begin{array}{l}\text { GetDamageModel () } \\
\text { Return a pointer to the damage model for this material. }\end{array}$ \\
\hline ShearModel * & $\begin{array}{l}\text { SetShearModel (const char *modelName) } \\
\text { Specify the type of shear model that this material should use. }\end{array}$ \\
\hline ShearModel * & $\begin{array}{l}\text { GetShearModel () } \\
\text { Return a pointer to the shear modulus model for this material. }\end{array}$ \\
\hline YieldSurfaceModel * & $\begin{array}{l}\text { SetYieldSurfaceModel (const char *modelName) } \\
\text { Specify the type of yield surface model that this material should use. }\end{array}$ \\
\hline YieldSurfaceModel * & $\begin{array}{l}\text { GetYieldSurfaceModel () } \\
\text { Return a pointer to the yield surface model for this material. }\end{array}$ \\
\hline const char * & $\begin{array}{l}\text { QueryEOSModelType () const } \\
\text { Returns the string name of the EOS model defined for this material. }\end{array}$ \\
\hline const char * & $\begin{array}{l}\text { QueryFlowStressModelType () const } \\
\text { Returns the string name of the flow stress model defined for this material. }\end{array}$ \\
\hline const char * & $\begin{array}{l}\text { QueryDamageModelType () const } \\
\text { Returns the string name of the damage model defined for this material. }\end{array}$ \\
\hline const char * & $\begin{array}{l}\text { QueryShearModelType () const } \\
\text { Returns the string name of the shear modulus model defined for this } \\
\text { material. }\end{array}$ \\
\hline const char * & QueryYieldSurfaceModelType () const \\
\hline
\end{tabular}


Returns the string name of the yield surface model defined for this material.

\title{
MatProp::ModelBase Class Reference
}

Base class for supporting a number of methods. Inheritance diagram for MatProp::ModelBase:

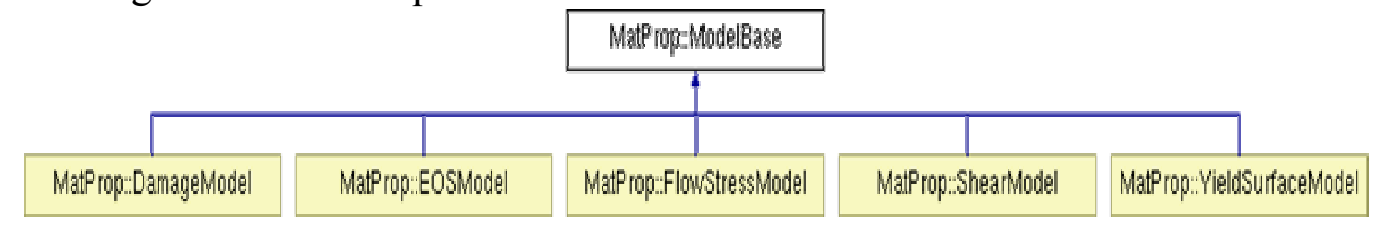

\author{
Public Member Functions \\ const char * GetModelTypeName () const \\ Returns the string that defines the model type. \\ void QueryParameterList (char ** \&parameters, int \&numParameters) \\ Return a list of the parameters supplied by this model. \\ void GetParameterCount (int \&numParameters) \\ Return the count of the parameter contained in the model. \\ void FreeParameterList (char ** \&parameters) const \\ Free resources (memory) associated with the parameter list query. \\ void QueryPolynomialList (char ** \&polynomials, int \&numPolynomials) \\ Returns an array of strings representing the available <polynomial>s to query \\ on this material. \\ void FreePolynomialList (char ** \&polynomials) const \\ Free resources (memory) associated with the parameter list query. \\ void GetPolynomialArray (const char *name, double *\&array, char ** \&names, int \\ \&size) const \\ Get a <polynomial> - coeficient names and values. \\ void FreePolynomialArray (double *\&array, char **\&names, const int size) \\ Free resources allocated by GetPolynomialArray. \\ double GetParameter (const char *name) const \\ Return a scalar <parameter> value. \\ int GetIntParameter (const char *name) const \\ Return an integer scalar <parameter $>$ value. \\ void GetParameter (const char *name, double \&value) const \\ Get a scalar <parameter> value (call by reference style). \\ void GetIntParameter (const char *name, int \&value) const \\ Get an integer scalar <parameter> value (call by reference style). \\ double GetPolynomialArrayEntry (const char *name, const char *entryName) const \\ Get a specific entry of a <polynomial> by name. \\ double GetPolynomialArrayEntry (const char *name, const int exponent) const \\ Get a specific entry of a <polynomial> by subscript. \\ bool SetUnits (Units units) \\ Set the unit based on the Group(1.1 Milestone). \\ bool SetUnit (UnitType type, char *val) \\ Set a specific unit type to a specific value(1.1 Milestone).
}




\section{Appendix C: Current Materials and Model Combinations.}

\begin{tabular}{|c|c|c|c|c|c|c|c|c|c|}
\hline \multicolumn{10}{|c|}{\begin{tabular}{|c|} 
Current Materials and Models \\
\end{tabular}} \\
\hline \multirow[b]{3}{*}{ Material Name } & \multicolumn{9}{|c|}{ Models } \\
\hline & \multicolumn{2}{|c|}{ eos } & \multicolumn{5}{|c|}{ flow_stress } & \multirow[b]{2}{*}{ 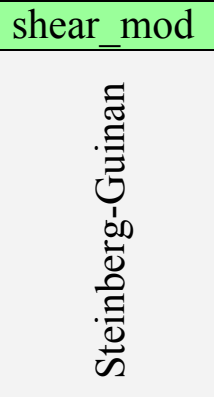 } & \multirow[b]{2}{*}{ 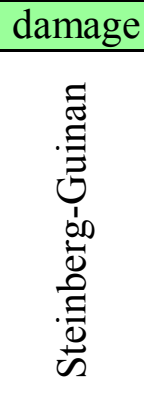 } \\
\hline & 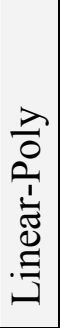 & 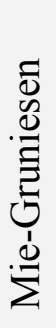 & $\vec{a}$ & $\stackrel{\mathscr{N}}{\Sigma}$ & 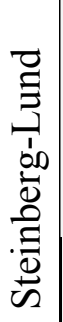 & 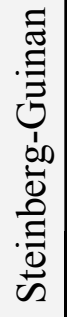 & $\begin{array}{l}y \\
0 \\
0 \\
0 \\
1 \\
0 \\
0 \\
0 \\
0 \\
0 \\
0\end{array}$ & & \\
\hline Aluminum 1100-O & * & * & & * & $*$ & * & * & * & * \\
\hline Aluminum 2024-T4 & & * & & & * & * & & $*$ & * \\
\hline Aluminum 6061-T6 & * & * & & . & * & * & * & * & * \\
\hline Beryllium & * & * & $*$ & * & * & * & & * & * \\
\hline Copper OFHC Half Hard & * & $*$ & & & * & * & * & * & * \\
\hline Gold & & $*$ & & & * & $*$ & & * & * \\
\hline Graphite & & * & & & * & * & & * & * \\
\hline Kel-F C2F3Cl & & * & & & * & $*$ & & * & * \\
\hline Lead & & $*$ & & & $*$ & $*$ & & * & * \\
\hline Lexan & & $*$ & & & * & $*$ & & * & * \\
\hline Lithium & & * & & & * & * & & * & * \\
\hline Lithium Fluoride & & $*$ & & & $*$ & * & & * & * \\
\hline Lucite & & $*$ & & & * & * & & * & * \\
\hline Mercury & & $*$ & & & * & * & & * & * \\
\hline Magnesium AZ31B & * & $*$ & & & $*$ & $*$ & & $*$ & * \\
\hline Micarta & & $*$ & & & $*$ & * & & $*$ & * \\
\hline Molybdenum & * & $*$ & $*$ & * & $*$ & $*$ & * & $*$ & * \\
\hline Monel 66\%Ni-29\%Cu-3\%Al-1/2\% & & * & & & * & * & & * & * \\
\hline $\begin{array}{l}\text { Fansteel } 85-61 \% \mathrm{Nb}-28 \% \mathrm{Ta}-10 \% \mathrm{~W} \\
-1 \% \mathrm{Zr}\end{array}$ & & $*$ & & & * & $*$ & & * & * \\
\hline Nickel & * & * & & & * & * & * & * & * \\
\hline Niobium & & * & & & * & * & & * & * \\
\hline Platinum & & * & & & * & * & & * & * \\
\hline Polypentene & & * & & & * & * & & * & * \\
\hline Silastic & & $*$ & & & * & * & & * & * \\
\hline Tantalum - 10\% Tungsten & * & * & & & * & * & & * & * \\
\hline Tantalum & * & * & $*$ & * & * & * & * & * & * \\
\hline Teflon & & * & & & * & * & & * & * \\
\hline Thorium & & * & & & * & * & & * & * \\
\hline Thulium & & $*$ & & & * & $*$ & & * & * \\
\hline Titanium $-6 \%$ Aluminum $-4 \%$ Vanadium & * & * & & & * & * & * & * & * \\
\hline
\end{tabular}




\begin{tabular}{|c|c|c|c|c|c|c|c|c|c|}
\hline \multicolumn{10}{|c|}{ Current Materials and Models(continued) } \\
\hline \multirow[b]{2}{*}{ Material Name } & \multicolumn{2}{|c|}{ eos } & \multicolumn{5}{|c|}{ flow_stress } & \multirow{2}{*}{ 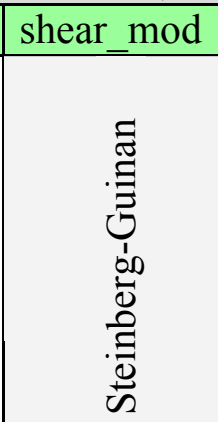 } & \multirow{2}{*}{ 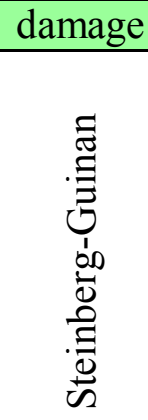 } \\
\hline & 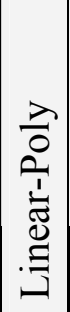 & 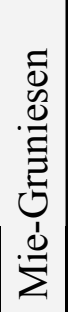 & $\vec{E}$ & $\stackrel{\mathscr{E}}{\dot{\Sigma}}$ & 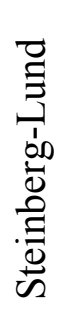 & 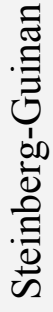 & 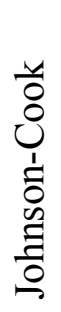 & & \\
\hline Tin & & * & & & * & * & & * & \\
\hline Titanium & & * & & & $*$ & * & * & * & * \\
\hline Tungsten & * & * & & * & * & * & * & * & * \\
\hline Tungsten Carbide & & $*$ & & & * & * & & * & * \\
\hline Uranium - $.75 \%$ Titanium & & * & & & * & * & * & * & * \\
\hline Uranium - 5\% Molybdenum & & * & & & $*$ & * & & * & * \\
\hline Uranium - $6 \%$ Noibium & & & & & & & * & & \\
\hline Uranium & * & * & * & & * & * & & * & * \\
\hline Vanadium & * & * & * & & * & * & & * & * \\
\hline Tungsten - $3.5 \%$ Nickel - 1.5\% Iron & & * & & & * & * & * & * & * \\
\hline Steel 4340 & * & & & & & & * & & \\
\hline Stainless Steel 304 & * & * & * & & $*$ & $*$ & & * & * \\
\hline Steel Vascomax 250 & & * & & & * & * & & * & * \\
\hline Stainless Steel 21-6-9 & & $*$ & $*$ & & $*$ & $*$ & * & $*$ & $*$ \\
\hline
\end{tabular}




\section{Appendix D: Current Material Properties and Model Parameters.}

\begin{tabular}{|c|l|l|}
\hline \multicolumn{2}{|c|}{ Material Properties } & \multicolumn{1}{|c|}{ Units* } \\
\hline Parameter & \multicolumn{1}{|c|}{ Description } & $\mathrm{g} / \mathrm{cm}^{3}$ \\
\hline rho0 & Reference density & $\mathrm{cm}^{3} / \mathrm{g}$ \\
\hline v0 & Reference (specific) volume & $\mathrm{eu} / \mathrm{g}$ \\
\hline $\mathrm{e} 0$ & Reference (internal) energy & $\mathrm{Mbar}$ \\
\hline pmin & Minimum pressure at which material fails & - \\
\hline bhe & HE parameter related to "volume" at the C-J condition & $\mathrm{g} / \mathrm{mole}$ \\
\hline ayz & Atomic weight & \multicolumn{2}{|c|}{} \\
\hline
\end{tabular}

\begin{tabular}{|c|c|c|c|}
\hline \multicolumn{4}{|c|}{ Material Models } \\
\hline Parameter & $\begin{array}{c}\text { Equation } \\
\text { variable }\end{array}$ & Description & Units* \\
\hline \multicolumn{4}{|c|}{ Model: Mie-Gruneisen Equation Of State } \\
\hline $\mathrm{c} 0$ & $\mathrm{C}_{0}$ & Bulk sound speed & $\mathrm{cm} / \mu \mathrm{s}$ \\
\hline s1 & $\mathrm{S}_{1}$ & Hugoniot linear slope coefficient & - \\
\hline $\mathrm{s} 2$ & $\mathrm{~S}_{2}$ & Hugoniot quadratic slope coefficient & - \\
\hline s3 & $\mathrm{S}_{3}$ & Hugoniot cubic slope coefficient & - \\
\hline gamma0 & $\gamma_{0}$ & Initial value of Gruneisen gamma & - \\
\hline $\mathrm{b}$ & $b$ & Compression dependence of $\gamma$ & - \\
\hline $\begin{array}{l}\text { ecxfit(ec0 } \\
\text {...ec9) }\end{array}$ & $\mathrm{E}_{\mathrm{i}}^{\mathrm{c}}$ & $\begin{array}{l}\text { Cold curve polynomial coefficients } \\
\text { (energy per reference volume) }\end{array}$ & $\mathrm{eu} / \mathrm{cm}^{3}$ \\
\hline $\begin{array}{l}\text { emxfit(em0 ... } \\
\text { em9) }\end{array}$ & $E^{m}$ & $\begin{array}{l}\text { Melt curve polynomial coefficients } \\
\text { (energy per reference volume) }\end{array}$ & $\mathrm{eu} / \mathrm{cm}^{3}$ \\
\hline etamin & & Minimum $\eta$ used in cold and melt curve calcs & - \\
\hline etamax & & Maximum $\eta$ used in cold and melt curve calcs & - \\
\hline \multicolumn{4}{|c|}{ Model: Steinberg-Guinan Shear Modulus } \\
\hline $\mathrm{cmu}$ & $\mathrm{G}_{0}$ & Reference shear modulus at $300 \mathrm{~K}$ & Mbar \\
\hline $\mathrm{au}$ & $\mathrm{a}$ & Pressure dependence of the shear modulus. & Mbar $^{-1}$ \\
\hline $\mathrm{bu}$ & $\mathrm{b}$ & Temperature dependence of the shear modulus. & $\mathrm{K}^{-\mathrm{I}}$ \\
\hline yp & $\mathrm{y}_{\mathrm{p}}$ & Parameter in melt transition. & - \\
\hline begr & & Minimum $\eta$ used in shear modulus calculation & - \\
\hline endr & & Maximum $\eta$ used in shear modulus calculation & - \\
\hline \multicolumn{4}{|c|}{ Model: Steinberg-Guinan Flow Stress } \\
\hline $\mathrm{y}$ & $\mathrm{Y}_{0}$ & Yield strength at Hugoniot elastic limit & Mbar \\
\hline $\mathrm{yb}$ & $\beta$ & Work hardening parameter & - \\
\hline yc & $\mathrm{n}$ & Work hardening exponent & - \\
\hline gam0 & $\varepsilon_{0}$ & Initial plastic strain & - \\
\hline ywhmx & $Y_{\max }$ & Work hardening maximum & Mbar \\
\hline
\end{tabular}




\begin{tabular}{|c|c|c|c|}
\hline \multicolumn{4}{|c|}{ Material Models } \\
\hline Parameter & $\begin{array}{l}\text { Equation } \\
\text { variable }\end{array}$ & Description & Units* \\
\hline $\mathrm{y}$ & $\mathrm{Y}_{0}$ & Yield strength at Hugoniot elastic limit & Mbar \\
\hline $\mathrm{yb}$ & $\beta$ & Work hardening parameter & - \\
\hline $\mathrm{yc}$ & $\mathrm{n}$ & \begin{tabular}{|l|} 
Work hardening exponent \\
\end{tabular} & - \\
\hline gam0 & $\varepsilon_{0}$ & Initial plastic strain & - \\
\hline ywhmx & $\mathrm{Y}_{\max }$ & \begin{tabular}{|l|} 
Work hardening maximum \\
\end{tabular} & Mbar \\
\hline $\mathrm{c} 1$ & $\mathrm{C}_{1}$ & Reference strain rate in thermal activation regime & $\mu \mathrm{s}^{-1}$ \\
\hline $\mathrm{c} 2$ & $\mathrm{C}_{2}$ & Coefficient in the drag regime & Mbar- $\mu \mathrm{s}$ \\
\hline uk & $\mathrm{U}_{\mathrm{k}}$ & Activation energy/over Boltzmann constant & $\mathrm{eV}$ \\
\hline ypeierls & $\mathrm{Y}_{\mathrm{p}}$ & Peierls stress & Mbar \\
\hline ya & $\mathrm{Y}_{\mathrm{A}}$ & Athermal yield strength & Mbar \\
\hline ystrmx & $\mathrm{Y}_{\text {max }}^{*}$ & Work hardening max for rate-dependent term & Mbar \\
\hline \multicolumn{4}{|c|}{ Model: Johnson-Cook Flow Stress } \\
\hline jc_a & A & Constant contribution to strength & Mbar \\
\hline jc $b$ & B & Coefficient for strain hardening & Mbar \\
\hline jc_c & $\mathrm{C}$ & Multiplier on the log strain rate term & - \\
\hline jc_m & $\mathrm{m}$ & Exponent on temperature term & - \\
\hline jc $n$ & $\mathrm{n}$ & Hardening exponent & - \\
\hline jc_p & $\mathrm{C}_{\mathrm{p}}$ & Linear pressure dependence multiplier & - \\
\hline tmelt & $\overline{T_{\text {melt }}}$ & Melt temperature & $\mathrm{K}$ \\
\hline troom & $T_{\text {room }}$ & Ambient room temperature & $\mathrm{K}$ \\
\hline epsd0 & $\dot{\varepsilon}_{0}$ & \begin{tabular}{|l|} 
Strain-rate normalization factor \\
\end{tabular} & $\mu \mathrm{s}^{-1}$ \\
\hline epsd_cut & - & Minimum strain rate used in evaluations & $\mu \mathrm{s}^{-1}$ \\
\hline \multicolumn{4}{|c|}{ Model: Steinberg-Guinan Damage } \\
\hline spall & & $\begin{array}{l}\text { Specifies that the material can experience spall. } \\
\text { When set to one (or non-zero), when the } \\
\text { maximum principal stress exceeds |pmin| the } \\
\text { material is defined to have spalled. }\end{array}$ & - \\
\hline dam0 & & Damage length? & $\mathrm{cm}$ \\
\hline pcrush & & Crush strength & Mbar \\
\hline \multicolumn{4}{|c|}{ Model: Preston-Tonks-Wallace (PTW) Flow Stress } \\
\hline molar_weight & $\mathrm{M}$ & Molar weight & $\mathrm{g} / \mathrm{mole}$ \\
\hline kappa & $\kappa$ & Scale factor for dimensionless temperature & - \\
\hline gamma & $\gamma$ & Scale factor for strain rate & - \\
\hline theta & $\theta$ & Initial hardening rate & - \\
\hline $\mathrm{p}$ & $\mathrm{p}$ & Hardening rate parameter & - \\
\hline s0 & $\mathrm{s}_{0}$ & Saturation stress at $0 \mathrm{~K}$ & - \\
\hline s_inf & $\mathrm{S}_{\infty}$ & Saturation stress at high temperature & - \\
\hline y0 & $\mathrm{y}_{0}$ & Yield strength at $0 \mathrm{~K}$ & - \\
\hline y_inf & $\mathrm{y}_{\infty}$ & Yield strength at high temperature & - \\
\hline y1 & $\mathrm{y}_{1}$ & Stress multiplier for yield model in drag regime & - \\
\hline $\mathrm{y} 2$ & $\mathrm{y}_{2}$ & Exponent for yield model in drag regime & - \\
\hline beta & $\beta$ & Exponent for saturation term in drag regime & - \\
\hline
\end{tabular}




\begin{tabular}{|c|c|c|c|}
\hline \multicolumn{4}{|c|}{ Material Models } \\
\hline Parameter & $\begin{array}{l}\text { Equation } \\
\text { variable }\end{array}$ & Description & Units* \\
\hline $\mathrm{g} 0$ & $\mathrm{G}_{0}$ & Shear modulus at $0 \mathrm{~K}$ & Mbar \\
\hline alpha & $\alpha$ & \begin{tabular}{|l|} 
Temperature dependent shear modulus factor \\
\end{tabular} & - \\
\hline tmelt & $\mathrm{T}_{\text {melt }}$ & Melt temperature & $\mathrm{K}$ \\
\hline epsd_cut & - & Minimum strain rate used in evaluations & $\mu \mathrm{s}^{-1}$ \\
\hline \multicolumn{4}{|c|}{ Model: Mechanical Threshold Stress (MTS) Flow Stress } \\
\hline kob3 & $\mathrm{k} / \mathrm{b}^{3}$ & $\mathrm{~b}=$ burgers vector, $\mathrm{k}=$ Boltzmann's constant & Mbar/K \\
\hline sig_a & $\hat{\sigma}_{a}$ & Athermal contribution to yield stress & Mbar \\
\hline sig_s & $\hat{\hat{\sigma}_{s}}$ & Threshold stress contribution from solute & Mbar \\
\hline $\mathrm{g} 0{ }_{-} \mathrm{s}$ & $\mathrm{g} 0 \mathrm{~s}_{\mathrm{s}}$ & Normalized activation energy: solute & - \\
\hline epsd_s & $\dot{\varepsilon}_{\mathrm{s}}$ & Base strain rate: solute & $\mu \mathrm{s}^{-1}$ \\
\hline q $\mathrm{s}$ & $\mathrm{q}_{\mathrm{s}}$ & Exponential in rate expression: solute & \\
\hline $\mathrm{ps}$ & $\mathrm{p}_{\mathrm{s}}$ & Exponential in rate expression: solute & \\
\hline sig_i & $\hat{\sigma}_{i}$ & \begin{tabular}{|l|} 
Threshold stress contribution from interstitial \\
\end{tabular} & Mbar \\
\hline g0_i & $\mathrm{gO}_{\mathrm{i}}$ & \begin{tabular}{|l|} 
Normalized activation energy: interstitial \\
\end{tabular} & - \\
\hline epsd_i & $\dot{\varepsilon}_{\mathrm{i}}$ & Base strain rate: interstitial & $\mu \mathrm{s}^{-1}$ \\
\hline $\mathrm{q}_{\mathrm{i}}^{\mathrm{i}}$ & $\overline{\mathrm{q}_{\mathrm{i}}}$ & Exponential in rate expression: interstitial & - \\
\hline $\mathrm{p}$ & $\mathrm{p}_{\mathrm{i}}$ & Exponential in rate expression: interstitial & - \\
\hline g0 eps & $\mathrm{g}_{0 \mathrm{~d}}$ & Normalized activation energy: dislocation & - \\
\hline epsd_eps & $\dot{\varepsilon}_{\mathrm{S}}$ & Base strain rate: dislocation & $\mu \mathrm{s}^{-1}$ \\
\hline q eps & $\mathrm{q}_{\mathrm{d}}$ & Exponential in rate expression: dislocation & - \\
\hline p eps & $p_{d}$ & Exponential in rate expression: dislocation & - \\
\hline sighat0 & $\hat{\sigma}_{s}$ & Initial vale of the evolving threshold stress & Mbar \\
\hline sig s0 & $\hat{\sigma}_{s 0}$ & Base value for saturation calculation & Mbar \\
\hline g0_eps_s & $\mathrm{g} 0_{\mathrm{ds}}$ or $\mathrm{A}$ & Normalized activation energy for saturation & $\mu \mathrm{s}^{-1}$ \\
\hline epsd eps $s$ & $\dot{\varepsilon}_{\mathrm{ds}}$ & Base strain rate for saturation expression & $\mu \mathrm{s}^{-1}$ \\
\hline hard form & - & Specify form for hardening evolution, $\mathrm{F}$. & - \\
\hline alpha & $\alpha$ & Coefficient in threshold stress evolution model & - \\
\hline hard power & $\xi$ & & - \\
\hline $\mathrm{cl}$ & $\mathrm{C}_{1}$ & Constant hardening rate coefficient & Mbar \\
\hline $\mathrm{c} 2$ & $\mathrm{C}_{2}$ & Coefficient on the logarithmic hardening rate & Mbar \\
\hline c3 & $\mathrm{C}_{3}$ & Coefficient on the parabolic hardening rate & Mbar \\
\hline $\mathrm{c} 4$ & $\mathrm{C}_{4}$ & Coefficient on the linear hardening rate & Mbar \\
\hline $\mathrm{c5}$ & $\mathrm{C}_{5}$ & $\begin{array}{l}\text { Coefficient on the rate/temperature dependent } \\
\text { hardening }\end{array}$ & Mbar \\
\hline c6 & $\mathrm{C}_{6}$ & $\begin{array}{l}\text { Temperature coefficient in exponent of } \\
\text { rate/temperature hardening }\end{array}$ & $\mathrm{K}^{-1}$ \\
\hline csat & $\mathrm{C}_{\text {sat }}$ & Coefficient for reducing saturation stress & - \\
\hline timeunit & $\tau$ & Time unit normalization ; 1.0 for $\mathrm{s}, 1 . \mathrm{e} 6$ for $\mu \mathrm{s}$ & $\mu \mathrm{s}$ \\
\hline epsd_cut & - & Minimum strain rate used in evaluations & $\mu \mathrm{s}^{-1}$ \\
\hline \multicolumn{4}{|c|}{ Model: Linear Polynomial EOS } \\
\hline $\mathrm{a} 0$ & $\mathrm{a}_{0}$ & Pressure at zero compression and energy & Mbar \\
\hline
\end{tabular}




\begin{tabular}{|l|l|l|l|}
\hline \multicolumn{1}{|c|}{ Parameter } & $\begin{array}{c}\text { Equation } \\
\text { variable }\end{array}$ & \multicolumn{1}{c|}{ Material Models } \\
\hline a1 & $\mathrm{a}_{1}$ & Description & \multicolumn{1}{c|}{ Units* } \\
\hline a2 & $\mathrm{a}_{2}$ & Quadratic compression term & Mbar \\
\hline a3 & $\mathrm{a}_{3}$ & Cubic compression term & Mbar \\
\hline b0 & $\mathrm{b}_{0}$ & Base Gruneisen coefficient, $\Gamma$ & Mbar \\
\hline b1 & $\mathrm{b}_{1}$ & Linear dependence of $\Gamma$ on compression & - \\
\hline b2 & $\mathrm{b}_{2}$ & Quadratic dependence of $\Gamma$ on compression & - \\
\hline
\end{tabular}

* The Units are the units stored specifically in the database. We will allow users the ability to retrieve the units from the database in different units if desired. The desired units must be compatible with the existing units (i.e. cm -> feet, or grams-> kilograms)

Model References

1. Maudlin, Davidson and Henninger, Implementation and Assessment of the MechanicalThreshold-Stress Model Using the EPIC2 and PINON Computer Codes, LA-11895-MS, Sept. 1990

2. Follasbee, P.S. and Kocks, U.F., A constitutive description of the deformation of copper based on the used of the mechanical threshold stress as an internal state variable, Acta Metall., 36(1) 81-93 (1988)

3. Steinberg, D.J., Cochran, S.G. and Guinan, M.W., A constitutive model for metals applicable at high-strain rate, J. Appl. Phys. 51(3) 1498-1504 (1980)

4. Steinberg, D.J. and Lund, C.M., A constitutive model for strain rates from $10^{-4}$ to $10^{6} \mathrm{~s}^{-1}$, J. Appl. Phys. 65(4) 1528-1533

5. Zerilli, F.J. and Armstrong, R.W., Dislocation-mechanics-based constitutive relations for material dynamics calculations, J. Appl. Phys. 61(5) 1816-1825 (1987)

6. Zerilli, F.J. and Armstrong, R.W., Description of tantalum deformation behavior by dislocation mechanics based constitutive relations, J. Appl. Phys. 68(4) 1580-1591 (1990)

7. Johnson, G.R. and Cook, W.H., A constitutive model and data for metals subjected to large strain, high strain rates and high temperatures, Proceedings of the Seventh International Symposium on Ballistics, The Hague, The Netherlands, pp. 541-547, April 1983

8. Preston, D.L., Tonks, D.L. and Wallace, D.C., Model of plastic deformation for extreme loading conditions, J. Appl. Phys. 93(1) 211-220 (2003)

9. Steinberg, D.J., Equation of state and strength properties of selected materials, UCRL-MA106439 (1996) 


\section{Appendix E: Abbreviated XML Database example We are currently working on units and unit conversion for the 1.1 release of the code. Many of the current parameters do not have their unit assigned as yet.}

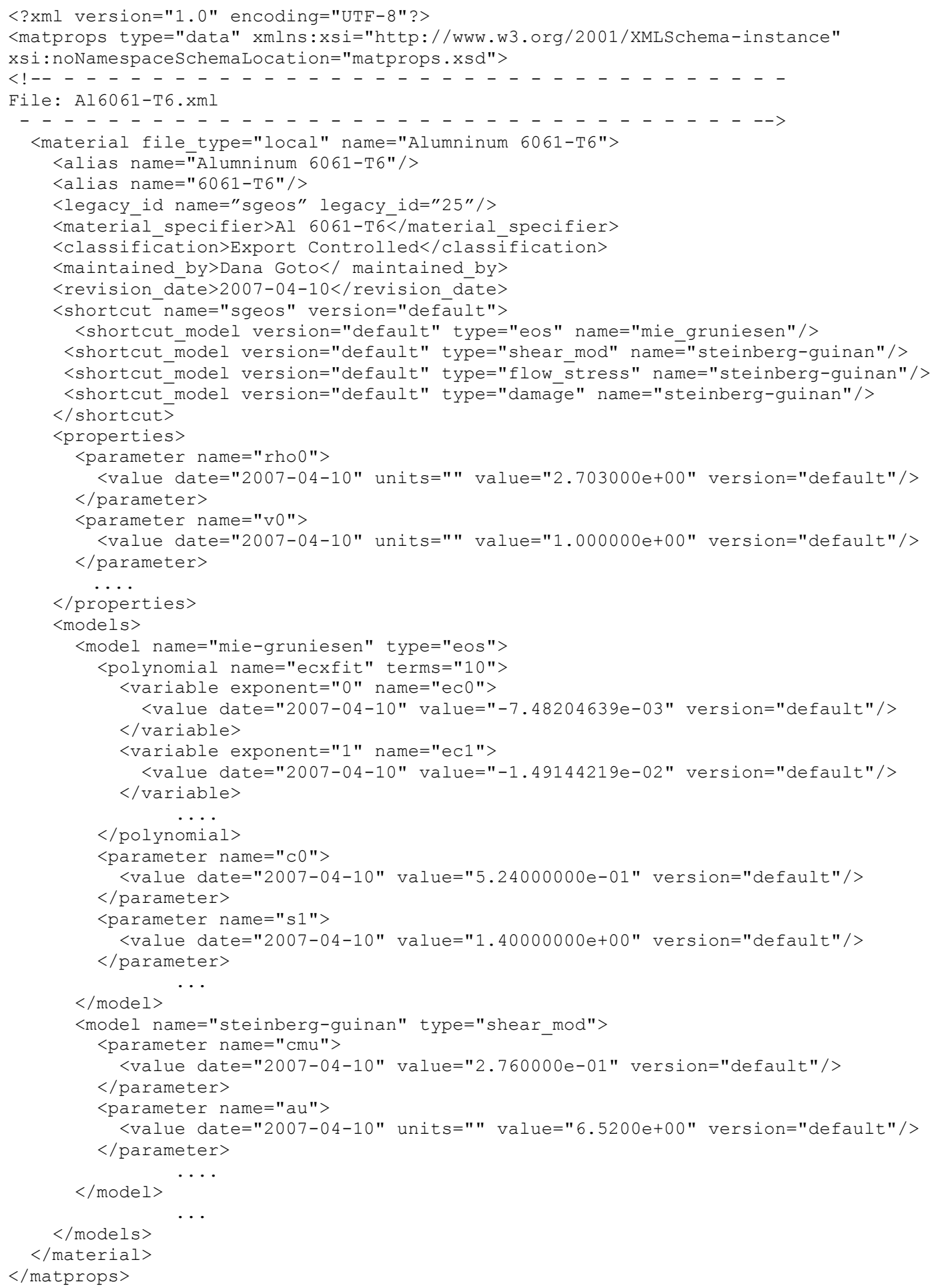

